\title{
Feedback flow control using the POD method on the backward facing step wall model
}

\author{
Sung-In Cho* and In Lee** \\ Division of Aerospace Engineering, School of Mechanical, Aerospace \& System Engineering, KAIST, 373-1 Guseong-dong, \\ Yuseong-gu, Daejeon 305-701, Korea
}

\author{
Seung-Jun Lee*** \\ Thermal-Hydraulics Safety Research Division, Korea Atomic Energy Research Institute, Daejeon, Republic of Korea
}

\author{
Choong Yun Lee ${ }^{* * * *}$ and Soo Hyung Park***** \\ Department of Aerospace Information Engineering, Konkuk University, Seoul, South Korea.
}

\begin{abstract}
Missiles suffer from flight instability problems at high angles of attack, since vortex flow over a fuselage cause lateral force to the body. To overcome this problem at a high angle of attack, the development of a real time vortex controller is needed. In this paper, Proper Orthogonal Decomposition (POD) and feedback controllers are developed for real time vortex control. The POD method is one of the most well known techniques for modeling low order models that represent the original full-order model. An adaptive control algorithm is used for real time control.
\end{abstract}

Key words: POD, ANN-ARX, OPD, real time feedback control

\section{Introduction}

Missiles suffer from flight instability problems at high angles of attack, since the vortex flow over a fuselage causes lateral force to the body [1]. To overcome this problem at high angles of attack, the development of a real time vortex controller is needed. For developing real time controllers, reduced order modeling techniques are surely necessary, since full simulation of the system is extremely time consuming. Aerodynamic forces can be determined by simple matrix algebra using the ROM technique. The POD method is one of the most well-known techniques for modeling low order models that represents the original full-order model
[2]. The basic idea of this method is to project N-dimensional state spaces onto an M-dimensional subspace, where $\mathrm{M}$ is much bigger than N. After the POD procedure, a system identification technique is performed to estimate POD modal amplitudes. An artificial Neural Network-Auto Regressive Exogenous (ANN-ARX) method, which estimates POD modal amplitude more accurately and faster than using Galerkin projection or stochastic estimation [3], is used in this paper. The reduced order model ( $\mathrm{ROM}$ ) is completed by organizing the POD method and an ANN-ARX model. After building a ROM, direct adaptive control law is chosen to close the loop. This method of control will help to reduce the vortex shedding and to achieve a desired closed loop flow state [4].
This is an Open Access article distributed under the terms of the Creative Commons Attribution Non-Commercial License (http://creativecommons.org/licenses/by$\mathrm{nc} / 3.0 /$ which permits unrestricted non-commercial use, distribution, and reproduction in any medium, provided the original work is properly cited. (c)

$$
\begin{aligned}
& \text { * Ph. D Student } \\
& \text { ** Professor, Corresponding author: inlee@kaist.ac.kr } \\
& \text { *** Ph. D researcher } \\
& * * * * \text { Ph. D Student }
\end{aligned}
$$$$
* * * * \text { Professor }
$$ 


\section{Reduced Order Modeling}

\subsection{Proper Orthogonal Decomposition (POD)}

POD is used to determine an optimal basis for representing a given data set. The goal of this method is to obtain an orthogonal basis, so that almost every member of the data can be decomposed relative to the basis. Therefore, the original higher order system can be represented by the combination of the minimum number of aerodynamic modes. That is, using the method, flow data, , which are functions of space and time, can be represented as follows [2] :

$$
\phi(X, t)=\sum_{j=1}^{M} a_{j}(t) \varphi_{j}(X)
$$

where $\varphi_{j}(X)$ is the POD mode and $a_{j}(t)$ is the POD modal amplitude. These can be represented in the matrix form as follows:

$$
\Phi(t)=\boldsymbol{\varphi} \mathbf{a}
$$

To obtain POD basis, $\varphi$, a snapshot method, is widely used for its convenience [5]. In this approach, full CFD simulation is performed initially. During this process, snapshots which have fluid information are recorded at selected times. Finally, a singular value decomposition of the snapshot matrix is performed to get POD modes.

\subsection{Artificial Neural Network-Auto Regressive Exog- enous (ANN-ARX)}

ANN-ARX is a system identification method which combines an artificial neural network and an auto-regressive exogenous model. It estimates POD modal amplitude more accurately and faster than using Galerkin projection or stochastic estimation [3]. The method can maximize the effectiveness of the real time feedback flow controller, since

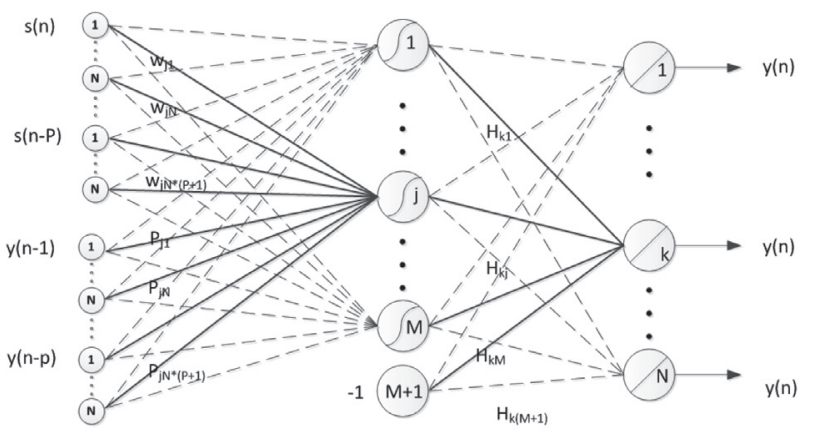

Fig. 1. Schematics of ANN-ARX model it excludes the CFD analysis for Navier-Stokes equation. The model was developed using a multi-layer perceptron, which contains more than 1 hidden layer, to increase the ability to estimate nonlinear behavior [6]. An ANN-ARX model was combined with the POD method for reduced order modeling of vortex flow. Schematics of the ANN-ARX model, which estimates POD modal amplitude, is shown in Fig. 1.

The density gradient, which was calculated by CFD analysis, was applied to the POD method to calculate POD mode and POD modal amplitude. ROM was completed by organizing the POD method and ANN-ARX model, which generates POD modal amplitudes. The flow chart is shown in Fig. 2.

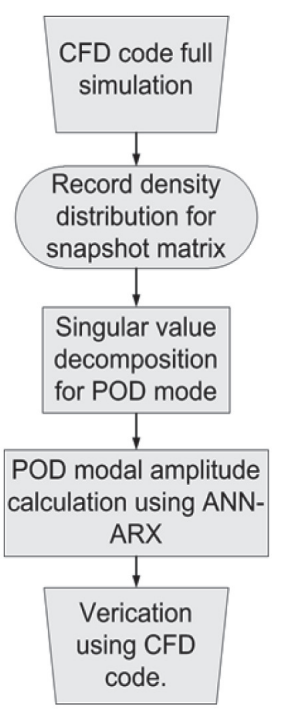

Fig. 2. Flow chart of reduced order modeling

\subsection{Direct model reference adaptive control}

Model reference control is based upon matching the response of a system to that of a reference model. The control system can be designed so that the inputs to the plant drive the outputs of the plant to follow the outputs of the model. A block diagram of the model reference control system is shown in Fig. 3.

The system for the continuous linear model reference control has the form [7]:

$$
\begin{aligned}
& \dot{x}_{p}(t)=A_{p} x_{p}(t)+B_{p} u_{p}(t) \\
& y_{p}(t)=C_{p} x_{p}(t)
\end{aligned}
$$

where $\dot{x}_{p}(t)$ is the plant state vector, $u_{p}(t)$ is the control vector, and $y_{p}(t)$ is the plant output vector. The objective 
of the model reference control is to find $u_{p}(t)$ so that the $y_{p}(t)$ approximates the output of the reference model which has the form:

$$
\begin{aligned}
& \dot{\mathrm{x}}_{\mathrm{m}}(\mathrm{t})=\mathrm{A}_{\mathrm{m}} \mathrm{x}_{\mathrm{m}}(\mathrm{t})+\mathrm{B}_{\mathrm{m}} \mathrm{u}_{\mathrm{m}}(\mathrm{t}) \\
& \mathrm{y}_{\mathrm{m}}(\mathrm{t})=\mathrm{C}_{\mathrm{m}} \mathrm{x}_{\mathrm{m}}(\mathrm{t})
\end{aligned}
$$

where $\dot{x}_{m}(t)$ is model state vector, $u_{m}(t)$ is the model input, and $y_{m}(t)$ is the model output vector.

When perfect output tracking occurs, the ideal plant which satisfies the same dynamics as the real plant can be written as linear functions of the model state and model input, which has the form:

$$
\begin{aligned}
& {\left[\begin{array}{c}
\dot{x}_{p}^{*}(t) \\
u_{p}^{*}(t)
\end{array}\right]=\left[\begin{array}{ll}
S_{11} & S_{12} \\
S_{21} & S_{22}
\end{array}\right]\left[\begin{array}{l}
x_{m}(t) \\
u_{m}
\end{array}\right]} \\
& {\left[\begin{array}{c}
\dot{x}_{p}^{*}(t) \\
y_{p}^{*}(t)
\end{array}\right]=\left[\begin{array}{cc}
A_{p} & B_{p} \\
C_{p} & 0
\end{array}\right]\left[\begin{array}{ll}
S_{11} & S_{12} \\
S_{21} & S_{22}
\end{array}\right]\left[\begin{array}{c}
x_{m}(t) \\
u_{m}
\end{array}\right]}
\end{aligned}
$$

where $x_{p}^{*}(t)$ and $u_{p}^{*}(t)$ denotes the ideal trajectories. From the equation $(3-6)$, the control input can be written as

$$
u_{p}(t)=u_{p}^{*}(t)+K\left(y_{m}-y_{p}\right)
$$

where $K$ is a stabilizing feedback gain. $u_{p}(t)$ was chosen as equation (7) to close the feedback loop for the wake control.

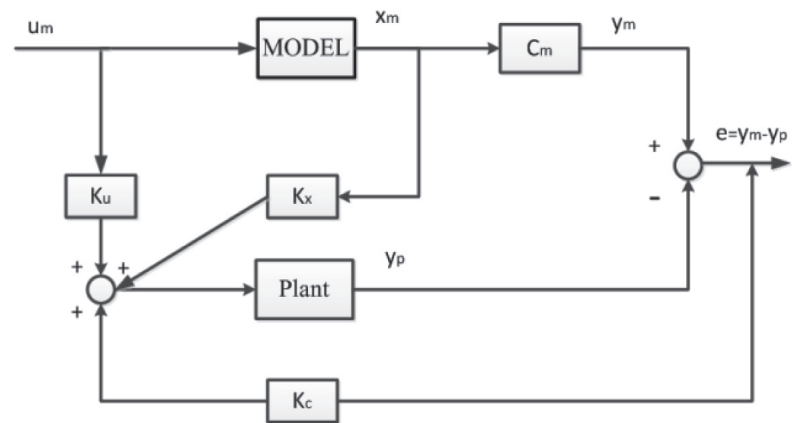

Fig. 3. Block diagram of the direct model reference adaptive controller

\section{Backward facing step wall model}

\subsection{Backward facing step wall}

The backward facing step wall model, which is the target model of this research, is shown in Fig. 4. A SpalartAllmaras turbulent model based on the DDES method was used to simulate the unsteady turbulent flow over a 3-D backward facing step [8]. The physical properties of the backward facing step wall model were the same as that of Driver and Seegmiller's experiment [9]. The height of the step is marked as and the heights of inlet and exit are $8 \mathrm{H}$ and $9 \mathrm{H}$, respectively. The isothermal viscous wall boundary condition is applied to the top and bottom wall, and the periodic boundary condition is applied in the z-direction. The inlet and outlet boundary were set as the specified turbulent boundary layer and the constant pressure outflow boundary condition, respectively. The properties of the model are represented in table 1.

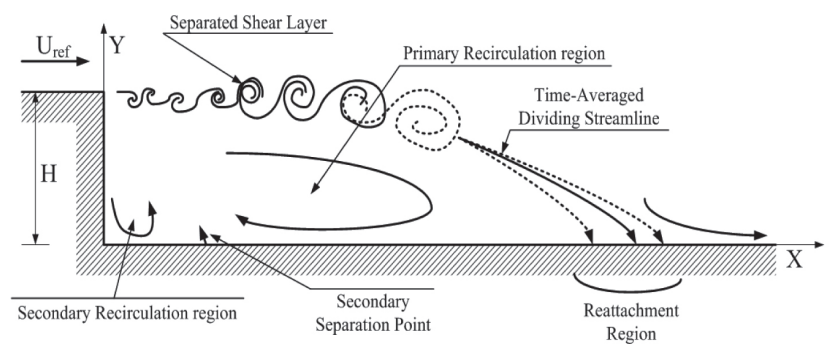

Fig. 4. Schematics of the wake model

Table 1. The properties of the backward facing step wall flow model

\begin{tabular}{ccc}
\hline Reynolds number & $\mathrm{Re}_{\mathrm{h}}$ & 38000 \\
Freestream velocity & $\mathrm{U}_{\infty}$ & $44.2 \mathrm{~m} / \mathrm{s}$ \\
Boundary layer thickness & $\delta$ & $1.9 \mathrm{~cm}$ \\
Step height & $\mathrm{H}$ & $1.27 \mathrm{~cm}$ \\
\hline
\end{tabular}

As shown in Fig. 5, the instantaneous vorticity contour displays small vortice structures breaking up from the shear layer. In order to build a flow database, a number of numerical simulations are performed with actuating amplitude and frequency for open-loop sinusoidal suctionblowing. Blowing and suction actuator was implemented at the edge of the step for open-loop control. The length of the actuator inlet is $0.08 \mathrm{H}$ and the actuator was installed parallel with the ground. The velocity, $\mathrm{U}_{\mathrm{a}}$ of the actuator was set as

$$
\mathrm{U}_{\mathrm{a}}=\mathrm{A}_{0} \sin (2 \pi \omega \mathrm{t})
$$

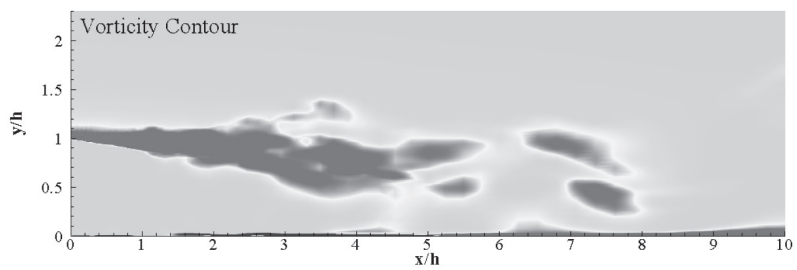

Fig. 5. Vorticity contour of CFD analysis 
where, $A_{0}$ is the amplitude and $\omega$ is the frequency of the actuator. The actuation frequencies of the open loop cases are determined as $200 \mathrm{~Hz}, 300 \mathrm{~Hz}$, and $400 \mathrm{~Hz}$. The actuation amplitudes are determined as $1 \%, 2 \%$ and $3 \%$ of the freestream velocity.

\subsection{ROM of the backward facing step wall}

To represent the extent of flow wake, the Optical Path Difference (OPD) was used in this research. Based on the Gladstone Dale relation, the Optical Path Length (OPL) was calculated to obtain the OPD [10]. Assuming that the optical beam is propagated in the $y$-direction, an average OPL in the $\mathrm{x}-\mathrm{z}$ plane is subtracted, and the OPD can be written as

$$
\mathrm{OPD}=\mathrm{OPL}-\overline{\mathrm{OPL}}^{\mathrm{x}, \mathrm{z}}
$$

where,

$$
\begin{aligned}
\mathrm{OPL} & =\int_{0}^{\mathrm{t}} \mathrm{n}(\mathrm{x}, \mathrm{t}) \mathrm{dx}=\int_{0}^{\mathrm{t}} \mathrm{K}_{\mathrm{GD}} \rho(\mathrm{x}, \mathrm{t}) \mathrm{dx} \\
\mathrm{K}_{\mathrm{GD}} & =2.23 \times 10^{-4}\left(1+\frac{7.52 \times 10^{-15}}{\lambda^{2}}\right)
\end{aligned}
$$

OPD is linearly dependent on the fluid density. Time history of OPD was arranged in a snapshot matrix and it was applied to the POD method to calculate POD mode and modal amplitude, as described in section 2.1. POD modes for all open loop cases were calculated, and the 1st POD modal amplitude for the baseline case is shown in the Fig. 6 .

Fig. 7 shows the comparison between the original CFD result and the reconstruction data using 2 POD modes. Openloop control case; and, was chosen for the comparison with CFD result and POD reconstruction. The results show good agreement between the two modes. From the results, it is verified that two POD modes are suitable for reduced order modeling.

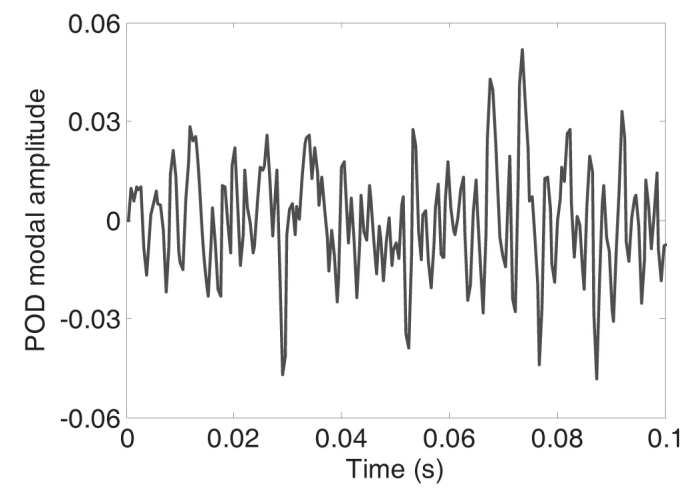

Fig. 6. POD 1st modal amplitude

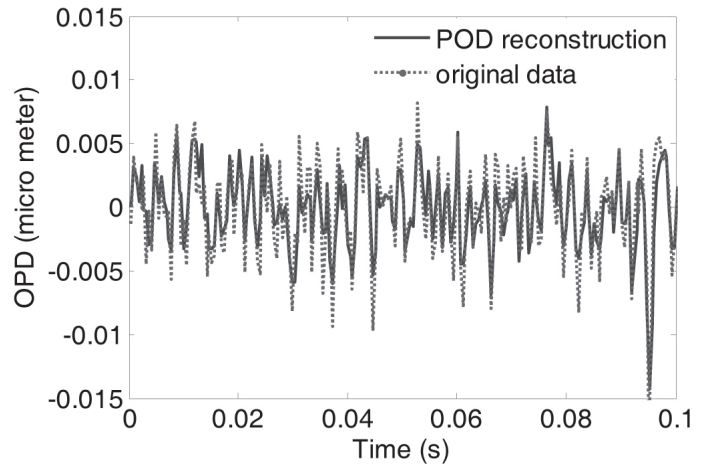

Fig. 7. OPD reconstruction data using two POD modes

ROM was completed by applying an ANN-ARX method to estimate POD modal amplitudes. At first, POD modal amplitude was calculated by the POD method and CFD results. Second, error back-propagation was applied for ANN-ARX modeling by appointing the actuator velocity as input, and the OPD data from sensors as output. Fig. 8 shows the OPD distribution among at an arbitrary time, and OPD sensors were placed at . After ANN-ARX estimates the OPD data from sensors, a mode estimator was constructed to estimate POD modal amplitude.

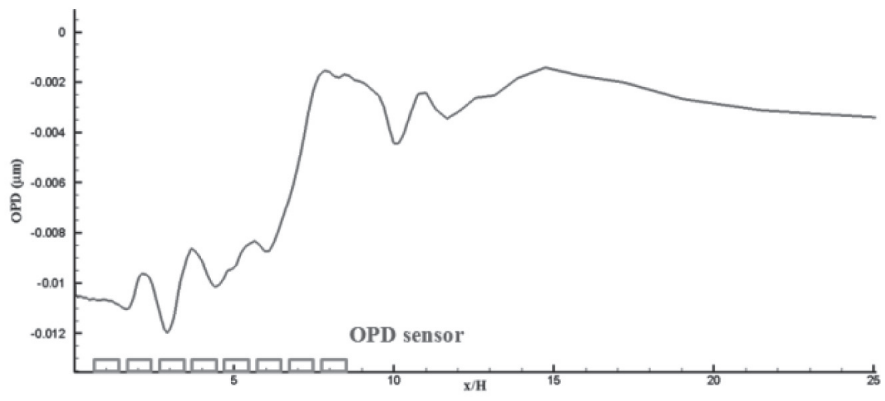

Fig. 8. OPD sensor distribution

The process which identifies the system using error backpropagation is called training, and the number of training cases is one of the most important parameters of the ROM. Training cases used in this study are shown in the table 2 . There are 10 cases depending on the amplitude and the frequency of the actuator, as represented in equation (3) including the baseline case. Nine cases were trained and one case was used as a validation case.

Table 2. Summary of cases. O: training cases, $\mathrm{X}$ : validation case

\begin{tabular}{cccc}
\hline & $\omega=200 \mathrm{~Hz}$ & $\omega=300 \mathrm{~Hz}$ & $\omega=400 \mathrm{~Hz}$ \\
\hline $\mathrm{A}_{0}=0.01$ & 0 & 0 & 0 \\
$\mathrm{~A}_{0}=0.02$ & 0 & 0 & $\mathrm{X}$ \\
$\mathrm{A}_{0}=0.03$ & 0 & 0 & 0 \\
\hline
\end{tabular}




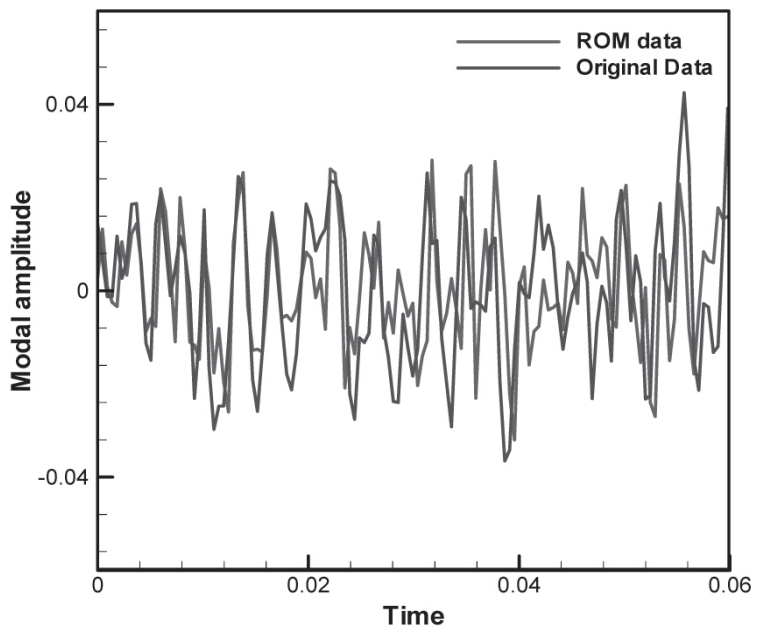

(a) $\mathrm{A}_{0} / \mathrm{U}_{\infty}=0.01, \omega=200 \mathrm{~Hz}$

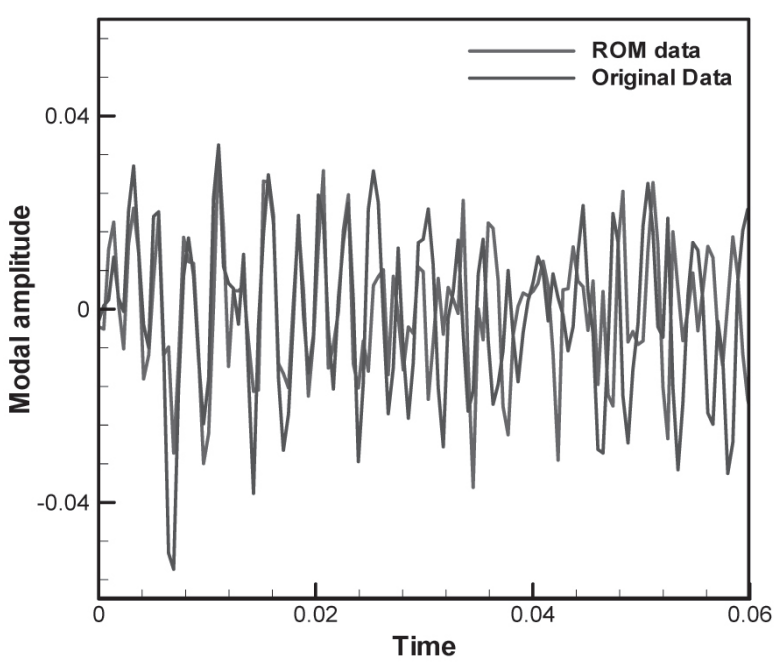

(c) $\mathrm{A}_{0} / \mathrm{U}_{\infty}=0.02, \omega=300 \mathrm{~Hz}$

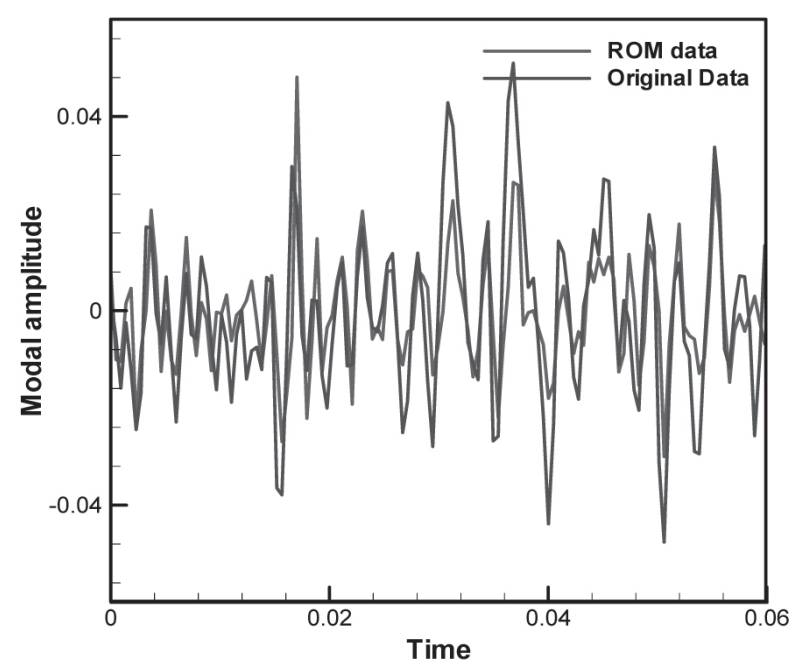

(e) $\mathrm{A}_{0} / \mathrm{U}_{\infty}=0.0$

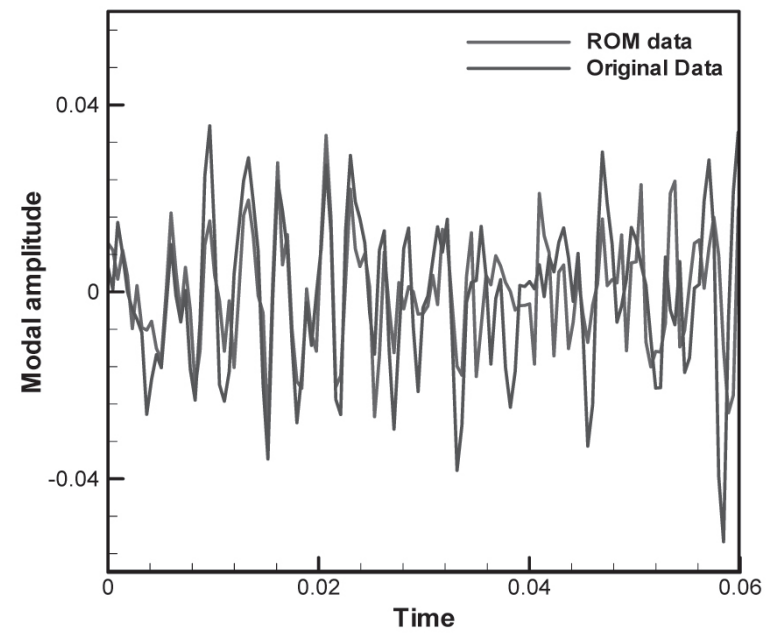

(b) $\mathrm{A}_{0} / \mathrm{U}_{\infty}=0.02, \omega=200 \mathrm{~Hz}$

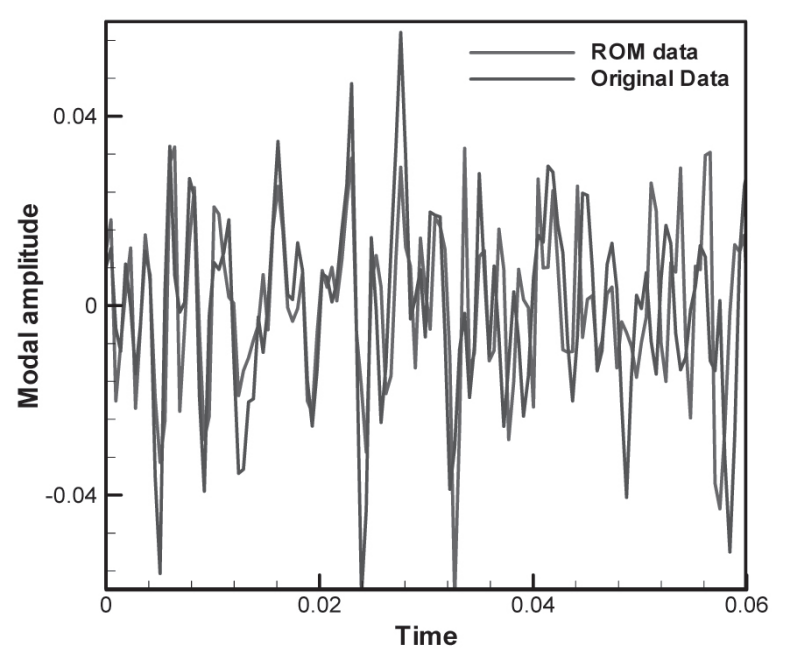

(d) $\mathrm{A}_{0} / \mathrm{U}_{\infty}=0.03, \omega=300 \mathrm{~Hz}$

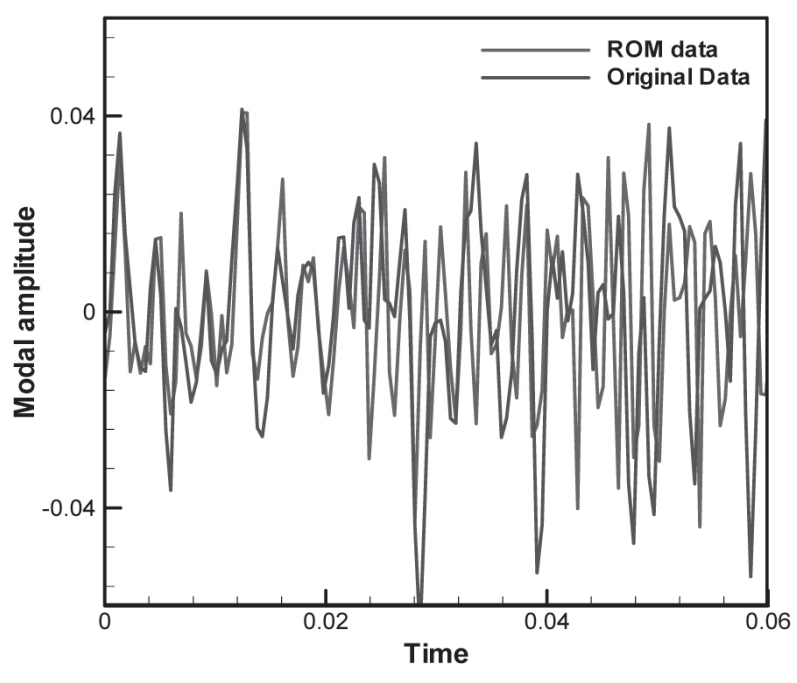

(f) $\mathrm{A}_{0} / \mathrm{U}_{\infty}=0.02, \omega=400 \mathrm{~Hz}$

Fig. 9. comparison of POD modal amplitude between the use of ANN-ARX and CFD analysis 
Fig. 9 shows the comparison of the results from the use of ANN-ARX and CFD analysis. Design cases and off-design cases were compared in Fig. 9. Fig. 9 (a) - (d) shows the open loop cases trained by specified amplitude and frequency, as represented in table 2. Fig. 9 (e) shows the baseline case and fig. 9 (f) shows the off-design case for validation. The figures show relatively good agreement between ANN-ARX and CFD analysis for the design cases, however, the error became larger for the off-design cases. From the results, the ROM model predicted the tendency of the CFD analysis, which is the most important precondition for real time feedback flow control using ROM models.

\subsection{Feedback flow controller}

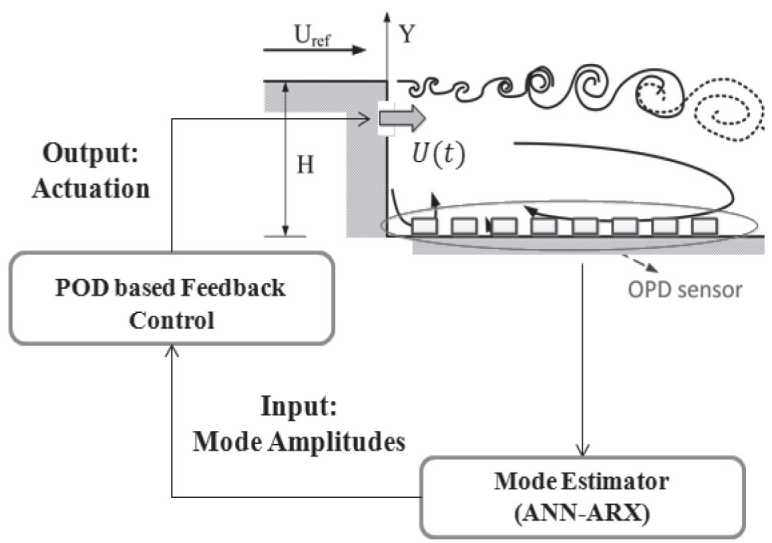

Fig. 10. control algorithm for the BFS model

ROM constructed by the POD and ANN-ARX methods was used for real time vortex control of the backward facing step wall model. A direct model reference adaptive controller was chosen for the feedback controller, and it was

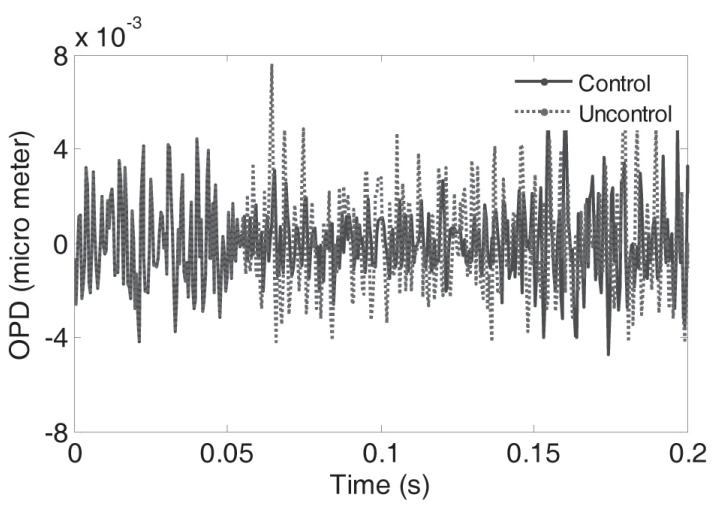

(a) designed to control the 1st POD modal amplitude, since it contains the greatest energy of the system. A block diagram of the controller and the schematics of the control model are shown in Fig. 10.

The control algorithm was applied to the ROM. Two POD modes were used for reconstruction of the wake flow. POD modal amplitudes were calculated when the baseline flow blows during $0 \sim 0.05 \mathrm{~s}$ and the controller actuated at 0.05 s. Finally, the controller was turned off and unforced flow redeveloped at $0.15 \mathrm{~s}$. The control results for POD modal amplitude are shown in Fig. 11. It shows that the direct model reference adaptive controller activated effectively when it was turned on. The OPD of the control results was reconstructed using two POD modal amplitudes and their spatial modes, as mentioned at section 3.2. The OPD at the point of interest, is plotted in Fig. 12 (a) and a spectral analysis of the OPD is plotted for the feedback flow control and uncontrolled case in Fig. 12 (b). The OPD variation shows that the OPD was reduced during the feedback control, and the dominant frequency of the feedback flow control signal corresponds to an uncontrolled signal.

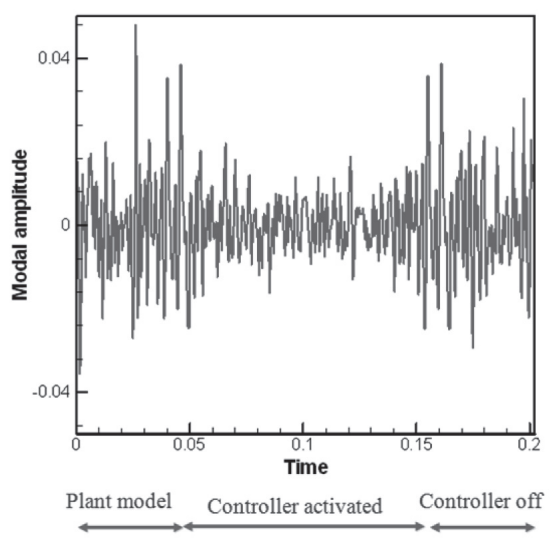

Fig. 11. Real time feedback flow control

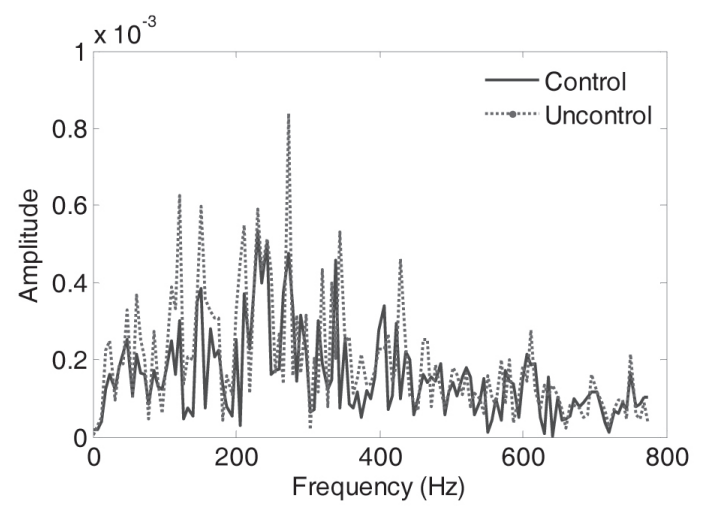

(b)

Fig. 12. OPD reconstruction of control data (a) and FFT result of OPD data (b) 


\section{Conclusion}

In this research, a Spalart-Allmaras turbulent model based DDES method was used to simulate the unsteady turbulent flow over a 3-D backward facing step. Since full simulation of the system is extremely time consuming, ROM were constructed by POD and ANN-ARX methods to develop a real time controller. Direct model reference adaptive control law was chosen to close the loop to reduce the vortex shedding, and to achieve a desired closed loop flow state. Designed feedback controller was used effectively to reduce the OPD of the BFS model, since the controller reduced the 1st POD modal amplitude of the wake flow.

\section{Acknowledgement}

The authors gratefully acknowledge the financial support provided by the Agency for Defense Development of Korea (UD100055CD). This research was also supported by the Basic Science Research Program through the National Research Foundation of Korea (NRF), funded by the Ministry of Education, Science and Technology(2010-0024136)

\section{References}

[1] Hamel, N., Corriveau, D., Wong, F., and Rainville, P.-A., "Numerical Investigation on the Leeside Vortex Manipulation of a Generic Missile", 23rd AIAA Applied Aerodynamics Conference, Toronto, Canada, 2005.
[2] Kwon, H. J., "Flutter Stability Prediction Using the Unsteady Aerodynamic Reduced-Order Model”, Ph.D. thesis, Division of Aerospace Engineering, Dept. of Mechanical Engineering, KAIST, 2003.

[3] Pindera, M. Z., "Adaptive flow control using simple artificial neural networks", 40th AIAA Aerospace Sciences Meeting \& Exhibit, Reno, Nevada, 2002.

[4] Siegel, S. G., Seide, J., Fagley, C., Luchtenburg, D. M., Cohen, K., and Mclaughlin, T., "Low-dimensional modelling of a transient cylinder wake using double proper orthogonal decomposition", Journal of Fluid Mechanics, Vol. 610, 2008, pp. 1-42.

[5] Sirovich. L., "Turbulence and the dynamics of coherent structures Part I: Coherent Structures", Applied Mathematics, Vol.45, No.3, 1987, pp. 561-571.

[6] Sahan, R.A., Koc-Sahan, N., Albin, D.C. and Liakopoulos, A., "Artificial neural network based modeling and intelligent control of transitional flows", Proceeding of 1997 IEEE International Conference on Control Applications, Hartford, Connecticut, 1997.

[7] Kaufman, H., Barkana, I., and Sobel, K., Direct Adaptive Control Algorithms Theory and Applications, 2nd Edition, Springer-Verlag New York Inc, New York, 1997.

[8] Lee, C. Y., Park, S. H, Cho, S. I., and Lee, I., "DelayedDetached Eddy Simulation for Optical Path Control on Backward-Facing Step Flow", Seventh International Conference on Computational Fluid Dynamics, Big Island, HI, 2012

[9] Driver, S. M., and Seegmiller, H. L., "Features of a Reattaching Turbulent Shear Layer in Divergent Channel Flow", AIAA Journal, Vol. 23, No. 2, 1985, pp. 163-171.

[10] Gilbert, K. G., and Otten, L. J., "Aero-Optical Phenomena", Progress in Astronautics and Aeronautics, Vol. 80, 1982, pp. 1-9. 